\title{
Lung cancer: biology and technology foster therapeutic innovation
}

On behalf of all authors, I am deeply honoured to welcome you to this special issue of Annals of Translational Medicine focusing on recent therapeutic breakthroughs in advanced lung cancer. As depicted in the title, the field of lung cancer has been an illustrative example of scientific progress through innovation, meaning that novel laboratory technologies have enabled the identification of molecular features of lung tumors that render them amenable to modern therapeutic strategies, such as newer chemotherapy regimens, molecular targeted agents and immunotherapy.

Lung cancer remains the leading cause of cancer related death worldwide, with approximately 1.6 million deaths recorded in 2015. Non-small cell lung cancer (NSCLC) accounts for approximately 80-85\% of cases. Despite broad advances in diagnostics and therapy, the 5-year overall survival for patients with advanced NSCLC has not significantly changed over the past few years. Following the decoding of human cancer genome and the advent of therapies targeting driver mutations, the choice of systemic therapy changed from "one size fits all" approach to a more precise selection of biologic therapies targeting distinct genetic profiles, the so-called "precision" medicine.

The first article of the volume (1) is a comprehensive overview of the emerging treatment landscape of NSCLC. It provides the background and the rational of how the advent of modern technologies for massive parallel DNA sequencing have elucidated the molecular aspects of NSCLC and have made possible to "dissect" the molecular anatomy of each individual tumor. This first chapter also introduces us to the recent therapeutic advances with the view to educate new practitioners and stimulate new thoughts for clinical trials.

The second article in this issue by Baxevanos et al. (2) concentrates on the recent advances in the field of chemotherapy by the introduction of newer agents such as nab-paclitaxel and nedaplatin and discusses the critical question as to whether chemotherapy regimens have reached a "plateau" in the treatment of advanced NSCLC, meaning that there is no room for further improvement of therapeutic outcomes by chemotherapy combinations, other than those of chemotherapy with either molecular agents or immunotherapy.

In a following article of this volume (3) the significant issue of oncogene addiction is addressed. More specifically, this article focuses on epidermal growth factor receptor (EGFR)-mutated NSCLC and discusses the recent breakthrough of the implementation of third-generation EGFR-tyrosine kinase inhibitors (EGFR-TKIs). The aim of this review is to describe the different treatment strategies that have been developed to tackle failure of first and second-line EGFR-TKIs, the corresponding mechanisms of resistance and the development of novel generation EGFR-TKIs that surpass acquired resistance. The challenge posed by the implementation of third-generation EGFR-TKIs earlier in the course of the disease in first-line treatment of EGFR-mutant NSCLC is also addressed.

Another example of oncogene-addicted NSCLC is anaplastic lymphoma kinase (ALK) positive NSCLC. Ziogas et al. (4) provide a comprehensive overview of ALK-rearranged NSCLC, emphasizing on the clinical development of ALK-TKIs, the problem of acquired resistance and the advent of second- and third-generation ALK inhibitors that possess the capacity to reverse resistance and render tumors amenable to ongoing ALK inhibition, providing thus substantial clinical benefit. They also discuss an important challenge, that of implementing newer ALK inhibitors in the first-line setting and provide data on the first studies directly comparing crizotinib with newer ALK inhibitors.

In the last few years, a growing body of evidence suggests that, beyond EGFR and ALK, a number of other, less frequent, but still clinically relevant genetic alterations can be therapeutically targeted in patients with heavily pretreated, advanced NSCLC. In the next article of this volume, Gkolfinopoulos (5) reports on the rare genetic alterations, including mutations in the genes KRAS, BRAF, HER2, PI3KCA and DDR2, but also ROS1 and RET rearrangements and MET, HER2 and FGFR1 gene amplifications.

Advanced squamous-cell lung cancer, used to be considered the "neglected sibling" of adenocarcinoma, due to the paucity of molecular targets and pertinent clinical trials for squamous histology. As shown in the article by Tsironis et al. (6) TCGA data and other studies using next-generation sequencing (NGS) have completely transformed the landscape, by identifying a number of potential therapeutic targets for squamous NSCLC. The authors also present recent therapeutic advances including molecular targeted agents and immunotherapy that are now an indispensable part of the therapeutic 
armamentarium against NSCLC with squamous histology.

A special volume dedicated to advanced lung cancer could not be complete without an extensive reference to the most fascinating aspect of modern therapeutics, which is undoubtedly immunotherapy. In their comprehensive review, Tsiara et al. (7) discuss in detail the evolution of immunotherapy against NSCLC, with a special emphasis on anti-programmed death 1 (PD1) and anti-programmed death ligand 1 (anti-PD-L1) monoclonal antibodies, that represent a huge therapeutic progress against this devastating disease. The authors provide data on all recent clinical trials in first- and second-line treatment of advanced NSCLC with single-agent immunotherapy (namely nivolumab, pembrolizumab, atezolizumab and durvalumab), immunotherapy combinations and combinations of chemotherapy with immunotherapy.

Small-cell lung cancer (SCLC) still represents $15 \%$ of all lung cancer cases and remains a devastating disease that bears a dismal prognosis; more importantly, therapeutic advanced for SCLC lag behind those reported for NSCLC. Tsoukalas et al. (8) discuss in detail the challenges posed by the unique molecular biology of SCLC and the recent therapeutic advances in the field, mainly in terms of early clinical trials evaluating a number of therapeutic strategies, such as molecular agents and immunotherapy or chemo-immunotherapy combinations.

Last but not least, in the final article of this issue (9), the same group of authors addresses the issue of neuroendocrine neoplasms of the lung besides SCLC, namely well-to-moderately differentiated neuroendocrine tumors (also known as carcinoids) and large cell neuroendocrine lung carcinoma, which account for approximately $4 \%$ of all lung neoplasms. Important issues like pathological taxonomy of lung neuroendocrine tumors, unique aspects of their molecular biology and clinical behavior, as well as therapeutic modalities and treatment strategies according to the specific tumor biology are critically discusses

Concluding, I wish to express my sincere gratitude to Annals of Translational Medicine for providing us this unique opportunity to cover such an important topic and especially all authors who contributed enthusiastically to this work. We certainly hope that this volume will be valuable to the readers of the journal and that it will stimulate fruitful thinking, useful discussions and dissemination of knowledge to the benefit of our patients.

\section{Acknowledgements}

None.

\section{References}

1. 1. Economopoulou P, Mountzios G. The emerging treatment landscape of advanced non-small cell lung cancer. Ann Transl Med 2018;6:138.

2. Baxevanos P, Mountzios G. Novel chemotherapy regimens for advanced lung cancer: have we reached a plateau? Ann Transl Med 2018;6:139.

3. Mountzios G. Making progress in epidermal growth factor receptor (EGFR)-mutant non-small cell lung cancer by surpassing resistance: third-generation EGFR tyrosine kinase inhibitors (EGFR-TKIs). Ann Transl Med 2018;6:140.

4. Ziogas DC, Tsiara A, Tsironis G, et al. Treating ALK positive non-small cell lung cancer. Ann Transl Med 2018;6:141.

5. Gkolfinopoulos S, Mountzios G. Beyond EGFR and ALK: targeting rare mutations in advanced non-small cell lung cancer. Ann Transl Med 2018;6:142.

6. Tsironis G, Ziogas DC, Kyriazoglou A, et al. Breakthroughs in the treatment of advanced squamous-cell NSCLC: not the neglected sibling anymore? Ann Transl Med 2018;6:143.

7. Tsiara A, Liontos M, Kaparelou M, et al. Implementation of immunotherapy in the treatment of advanced non-small cell lung cancer (NSCLC). Ann Transl Med 2018;6:144.

8. Tsoukalas N, Aravantinou-Fatorou E, Baxevanos P, et al. Advanced small cell lung cancer (SCLC): new challenges and new expectations. Ann Transl Med 2018;6:145.

9. Tsoukalas N, Baxevanos P, Aravantinou-Fatorou E, et al. Advances on systemic treatment for lung neuroendocrine neoplasms. Ann Transl Med 2018;6:146. 


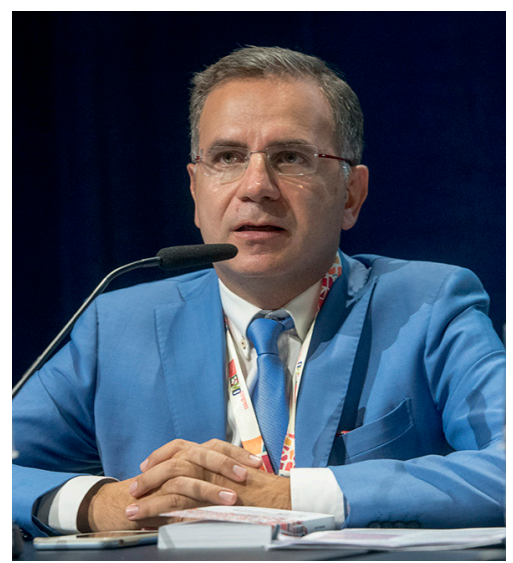

Giannis Mountzios

Giannis Mountzios ${ }^{1,2}$

${ }^{1}$ Department of Medical Oncology and Translational Research Athens, University of Athens School of Medicine, Athens, Greece; ${ }^{2}$ Second Department of Medical Oncology, Henry Dunant Hospital Center, Athens, Greece. (Email: gmountzios@gmail.com) doi: 10.21037/atm.2018.04.19

Conflicts of Interest: The author has no conflicts of interest to declare. View this article at: http://dx.doi.org/10.21037/atm.2018.04.19

Cite this article as: Mountzios G. Lung cancer: biology and technology foster therapeutic innovation. Ann Transl Med 2018;6(8):137. doi: 10.21037/atm.2018.04.19 